\title{
Expansão rápida da maxila - avaliação de dois métodos de reconstrução 3D por meio de um modelo laboratorial
}

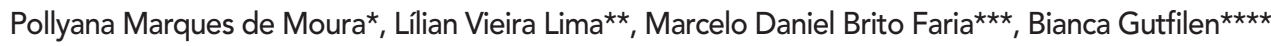

\begin{abstract}
Resumo
Objetivo: a proposta deste artigo é apresentar um modelo laboratorial para aquisição de imagens tomográficas para avaliação tridimensional do procedimento de expansão rápida da maxila (ERM). Métodos: o procedimento de expansão rápida da maxila foi realizado em crânio seco e os exames foram executados em tomógrafo de alta resolução e em tomógrafo de feixe cônico, antes e depois da ERM. Resultados: as imagens obtidas a partir do crânio seco, tomografia computadorizada multislice (TC) e tomografia volumétrica (CBCT) produziram resultados semelhantes para avaliação da abertura da sutura palatina mediana. Conclusão: a utilização de cortes tomográficos, adquiridos tanto por meio de tomógrafo computadorizado de alta resolução quanto por meio da técnica cone beam, possibilitou a reconstrução 3D da maxila com objetivo de oferecer a real avaliação da abertura intermaxilar.
\end{abstract}

Palavras-chave: Diagnóstico por imagem. Tomografia. Expansão rápida da maxila. Crânio seco.

\section{INTRODUÇÃO}

O conceito da reconstrução em três dimensões (3D), na área da Saúde, existe bem antes da descoberta do aparelho de raios $\mathrm{X}$ e pode ser demonstrado através de modelos em cera do século XVIII e XIX, que apresentam os aspectos anatômicos e patológicos dos órgãos do corpo humano ${ }^{11}$. A reconstrução em 3D por imagens permite melhor visualização das estruturas anatômicas, que normalmente se sobrepõem nas radiografias convencionais ${ }^{9}$.

O processo da reconstrução tridimensional inicia-se a partir da secção da região a ser analisada, por meio de um tomógrafo computadorizado, resultando na síntese destes cortes por intermédio de programas de computadores, permitindo uma visualização espacial do objeto estudado. No entanto, apesar da tomografia computadorizada (TC) oferecer um melhor delineamento das estruturas ósseas da face do que a radiografia convencional, o alto custo dos exames e as altas doses de radiação exigem que o ortodontista selecione as situações em que os benefícios são superiores aos riscos ${ }^{10}$.

A técnica cone beam computed tomography (CBCT) vem sendo introduzida na literatura odontológica como uma inovação na maneira de adquirir imagens volumétricas, com posterior reconstrução multiplanar. As mais importantes características são a menor quantidade de radiação e a alta resolução

* Especialista em Ortodontia FO-UERJ e mestranda em Ciências (Radiologia) FM-UFRJ.

** Especialista em Radiologia FO-UFRJ.

*** Professor adjunto de Radiologia FO-UFRJ.

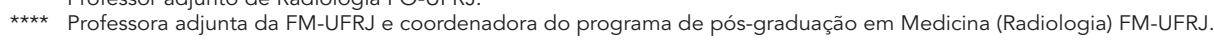


das imagens das estruturas dentomaxilofaciais, quando comparadas às da técnica fan bean ${ }^{13}$. De fato, o tomógrafo computadorizado multislice é um sistema grande e caro, designado primeiramente para exame de todo o corpo. O tomógrafo $\mathrm{CBCT}$ apresenta-se como um sistema menor, com menor custo, desenhado para aquisição de imagens do complexo maxilomandibular? .

A proposta deste artigo é apresentar um modelo laboratorial para avaliação tridimensional do procedimento de expansão rápida da maxila (ERM) por meio de tomógrafo de alta resolução e tomógrafo de feixe cônico.

\section{MATERIAL E MÉTODOS}

Neste estudo, um modelo laboratorial para análise do efeito transverso da ERM, por meio de diferentes recursos de reconstrução tridimensional, foi desenvolvido a partir de um crânio seco humano pertencente à disciplina de Radiologia da Faculdade de Odontologia da Universidade Federal do Rio de Janeiro (FO/UFRJ).

A expansão maxilar foi realizada com um disjuntor tipo Haas, com anéis adaptados aos segundos molares e aos primeiros pré-molares, pois esses elementos apresentavam suas raízes com maior inserção no processo alveolar, oferecendo melhor ancoragem para realização do procedimento.

Para viabilizar a abertura do parafuso expansor (Dentaurum, 600-301, com capacidade para 7,5mm de expansão), por tratar-se de um crânio seco de um adulto com maturidade esquelética, uma lima para gesso foi utilizada para romper a interdigitação da sutura palatina mediana, desde a espinha nasal anterior até o ponto de interseção da sutura palatina mediana e sutura palatina transversa. Em seguida, o parafuso foi acionado 4 voltas completas, com uma abertura linear de $3,2 \mathrm{~mm}$, ponto a partir do qual a resistência óssea era suficiente para deformar a chave de ativação do parafuso, não permitindo uma abertura maior.

Para a obtenção das TC, o aparelho Asteion ${ }^{\mathrm{TM}}$ (Toshiba, Tokyo, Japan) foi configurado com: $120 \mathrm{Kvp}, 80 \mathrm{~mA}$, cortes de $1 \mathrm{~mm}$ de espessura e $1 \mathrm{~mm}$ de intervalo, e tempo de exposição de 3,6s. As CBCT foram obtidas através do iCAT ${ }^{\mathrm{TM}}$ (Imaging Sciences International, Hatfield, PA), configurado com $120 \mathrm{Kvp}, 30 \mathrm{~mA}$, cortes de $1 \mathrm{~mm}$ e voxel de $0,4 \mathrm{~mm}$. A reconstrução tridimensional das estruturas maxilares foi realizada pelo programa Dental Slice V. 2.1 (BioParts Brasília/DF, Brasil) para todos os exames tomográficos.
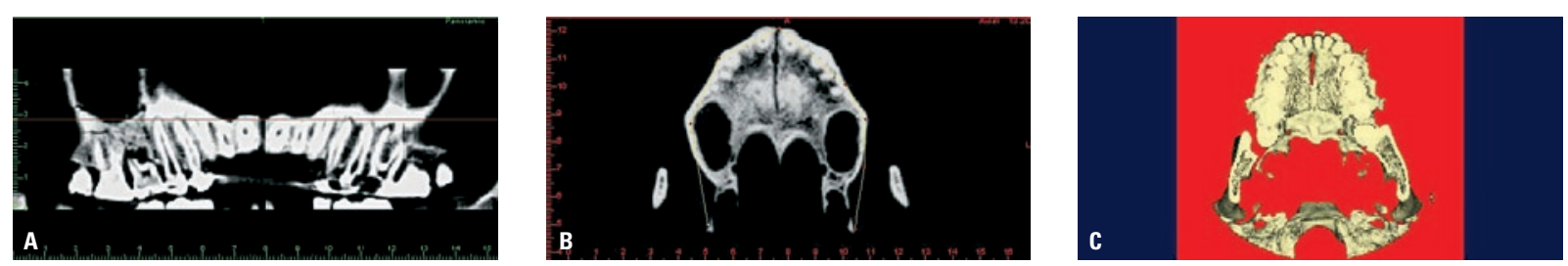

FIGURA 1 - Imagens obtidas por meio do iCAT ${ }^{\mathrm{TM}}$ 3-D Dental Imaging System após a expansão da maxila. Observa-se a linha de corte axial ao nível dos ápices dos pré-molares em: A) vista panorâmica, B) corte axial, C) reconstrução 3D com plano de corte axial.
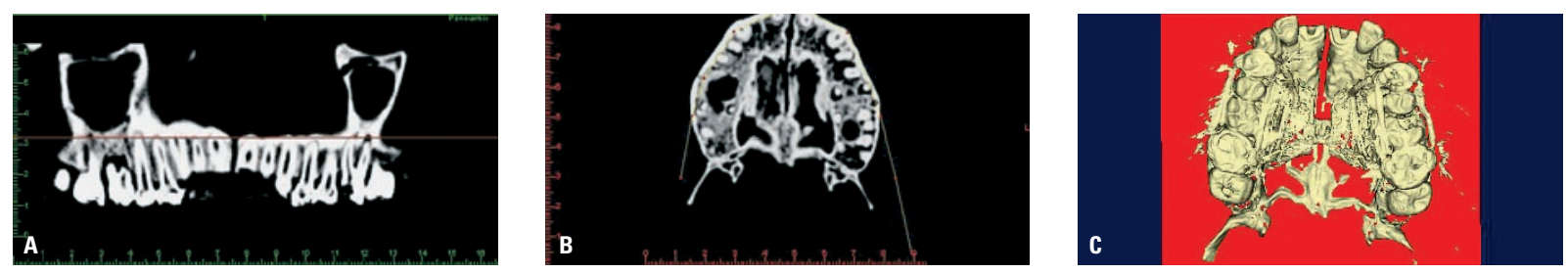

FIGURA 2 - Imagens obtidas por meio do Asteion ${ }^{T M}$-Toshiba após a expansão da maxila. Observa-se a linha de corte axial ao nível dos ápices dos pré-molares em: A) vista panorâmica, B) corte axial e C) reconstrução 3D com plano de corte axial. 


\section{RESULTADOS}

Os cortes foram realizados ao nível das raízes dos pré-molares para avaliação axial; nessa profundidade de corte, não se observavam artefatos produzidos pelo aparelho expansor. A aquisição das imagens produziu resultados semelhantes para avaliação da abertura da sutura palatina mediana por uma e outra técnica (Fig. 1, 2).

\section{DISCUSSÃO}

A evidência clínica de separação dos processos maxilares após a ERM é percebida pela abertura gradual do diastema entre os incisivos centrais superiores. $\mathrm{O}$ instrumento de diagnóstico utilizado usualmente para evidenciar a disjunção da sutura palatina mediana é a radiografia oclusal total da maxila ${ }^{1,2,3,4}$ que é, por vezes, ineficaz, por apresentar estruturas tridimensionais em duas dimensões. Assim, os limites da ERM são determinados eminentemente por critérios clínicos, havendo a pausa da ativação quando há o contato das cúspides palatinas dos molares superiores com as cúspides vestibulares dos molares inferiores ${ }^{2,3}$.

Somente a expansão proveniente das bases maxilares através da expansão sutural permanece estável ${ }^{2,10}$. De fato, a obtenção de um efeito limitado na base óssea pode implicar em restrições no resultado da terapia, o que suscita a busca por meios de diagnóstico por imagem que permitam avaliar o real grau de abertura intermaxilar.

Garib et al. ${ }^{5}$ compararam os efeitos dentoesqueléticos da ERM por meio de imagem coronal de $\mathrm{CT}$, sugerindo que essa abordagem, por sua precisão, pode ser comparada aos estudos com implantes metálicos. De fato, o uso de CT permite medições na dimensão transversa da maxila, bem como as mudanças na inclinação dos dentes posteriores, fazendo uma reprodução real da maxila nos três planos do espaço. No entanto, as dificuldades da realização de estudos com CT para avaliação dos efeitos da ERM vão desde a grande exposição de grupos de humanos à radiação (para efeito de pesquisa) à necessidade de remoção do aparelho expansor no momento da tomada do exame (para que se minimize a produção de artefatos) e seu custo financeiro ${ }^{5,10}$.

A CBCT parece estar indicada para estudar em 3D os efeitos da ERM, pois a aquisição volumétrica das imagens expõe o indivíduo a uma menor dose de radiação e a presença de detector específico para osso e dente faz com que a técnica cone beam otimize a utilização de raios $\mathrm{X}$, quando comparada à fan beam ${ }^{8,12}$. As imagens que ilustram esse artigo sugerem que tanto um quanto o outro exame podem ser utilizados para avaliação do procedimento de ERM, contudo há necessidade de uma amostra maior para a confirmação desses dados, possibilitando discorrer sobre qual técnica é melhor, quanto à visualização, nitidez e ausência de artefatos.

A utilização de crânios secos possibilita a comparação de diferentes técnicas tomográficas sem a exposição de pacientes a doses de radiação, por isso é apresentada como alternativa para o estudo dos efeitos da ERM em 3D. Jafari, Shetty e Kumar ${ }^{6}$ utilizaram o crânio seco de uma menina de 12 anos de idade para analisar os efeitos da ERM, por meio de elementos finitos, comprovando a eficácia do método. Lascala, Panella e Marques ${ }^{7}$ analisaram a precisão de medidas lineares obtidas por CBCT em crânio seco e verificaram que as medidas tomadas em estruturas dentomaxilofaciais foram similares às medidas reais, mas as medidas na base do crânio foram menores do que as realizadas nos crânios secos.

A utilização de reconstruções tridimensionais possibilita averiguar, ainda com o aparelho expansor em posição, qual a real abertura conseguida e fazer a sobrecorreção necessária para o procedimento, baseando-se em mensurações da base óssea. A imagem tridimensional do arco dentário pode ser usada como modelo de estudo, dispensando-se a moldagem tradicional, difícil de ser realizada com o aparelho em posição, principalmente após as expansões assistidas cirurgicamente.

\section{CONCLUSÃO}

A utilização de cortes tomográficos, adquiridos 
tanto por meio de tomógrafo computadorizado de alta resolução quanto por meio da técnica cone beam, possibilita a reconstrução tridimensional da maxila após o procedimento de expansão rápida, com propósito de possibilitar uma real avaliação da abertura intermaxilar.

\title{
Rapid maxillary expansion - evaluation of two methods of 3D reconstruction by means of a laboratorial model
}

\begin{abstract}
Objective: This paper proposes to present a laboratorial model for acquisition of tomographic images for threedimensional evaluation of the rapid maxillary expansion procedure. Methods: The procedure of rapid maxillary expansion was developed using a dry skull and the images were acquired from a multi-slice tomography and a volumetric tomography. The examinations were taken prior to and after the ERM. Results: The images acquired from the skull, multislice computed tomography (CT) and cone beam computed tomography (CBCT) had produced similar results. Conclusion: The use of tomographic slices, acquired by means of high resolution computerized tomography, as well as the cone beam technique, make possible the three-dimensional reconstruction of the palatomaxillary region in order to offer the real evaluation of the sutural opening.
\end{abstract}

Key words: Image diagnostics. Tomography. Rapid maxillary expansion. Dry skull.

\section{REFERÊNCIAS}

1. BACCETTI, T.; FRANCHI, L.; CAMERON, C. G.; McNAMARA JR., J. A. Treatment timing for rapid maxillary expansion. Angle Orthod., Appleton, v. 71, no. 5, p. 343-350, Oct. 2001.

2. CAPELOZZA FILHO, L.; CARDOSO NETO, J.; SILVA FILHO, O. G.; URSI, W. J. Non-surgically assisted rapid maxillary expansion in adults. Int. J. Adult Orthodon. Orthognath. Surg., Chicago, v. 11, no. 1, p. 57-66, 1996.

3. CAPELOZZA FILHO, L.; SILVA FILHO, O. G. Expansão rápida da maxila: considerações gerais e aplicação clínica. Parte I. Rev. Dental Press Ortodon. Ortop. Facial, Maringá, v. 2, n. 3, p. 88-101, maio/jun. 1997.

4. CAPELOZZA FILHO, L.; SILVA FILHO, O. G. Expansão rápida da maxila: considerações gerais e aplicação clínica. Parte II. Rev. Dental Press Ortodon. Ortop. Facial, Maringá, v. 2, n. 4, p. 86-108, jul/ago. 1997.

5. GARIB, D. G.; HENRIQUES, J. F. C.; JANSON, G.; FREITAS, M. R.; COELHO, R. A. Rapid maxillary expansion - tooth tissueborne versus tooth-borne expanders: a computed tomography evaluation of dentoskeletal effects. Angle Orthod., Appleton, v. 75, no. 4, p. 548-557, 2005.

6. JAFARI, A.; SHETTY, K. S.; KUMAR, M. Study of stress distribution and displacement of various craniofacial structures following application of transverse orthopedic forces: a threedimensional FEM study. Angle Orthod., Appleton, v. 73, no. 1, p. 12-20, Feb. 2003

7. LASCALA, C. A.; PANELLA, J.; MARQUES, M. M. Analysis of the accuracy of linear measurements obtained by cone beam computed tomography (CBCT - NewTom). Dentomaxillofac. Radiol., Stanford, v. 33, no. 5, p. 291-294, Sept. 2004.

8. MAKI, K.; INOU, N.; TAKANOSHI, A.; MILLER, A. J. Computer-assisted simulations in orthodontic diagnosis and the application of a new cone beam X-ray computed tomography. Orthod. Craniofac., Oxford, v. 6, no. 1, p. 95-101, 2003.
9. RIBEIRO-ROTTA, R. J. Técnicas tomográficas aplicadas à Ortodontia: a evolução do diagnóstico por imagens. Rev. Clin. Ortodon. Dental Press, Maringá, v. 9, n. 5, p. 102-156, set./ out. 2004.

10. RIZZATTO, S. M. D.; THIESEN, G.; REGO, M. V. N. N.; MARCHIORO, E. M.; MENEZES, L. M.; HOEFEL, J. R.; VIEIRA, J. F. Avaliando o procedimento da expansão rápida da maxila através da tomografia computadorizada helicoidal. Rev. Clin. Ortodon. Dental Press, Maringá, v. 3, n. 4, p. 76-87, ago./set. 2004.

11. SANNOMIYA, E. K.; FURUKAWA, S.; CASTILHO, J. C. M.; GONÇALVES, M. Utilização de reconstrução de imagens em três dimensões na Odontologia: uma nova era? Rev. Bras. Odontol., Rio de Janeiro, v. 58, n. 2, p. 105-107, mar./abr. 2001

12. SUKOVIC, P. Cone beam computed tomography in craniofacial imaging. Orthod. Craniofac. Res., Oxford, v. 6, no. 1, p. 31-36, 2003.

13. TOGASHI, K.; KITUARA, H.; YONETSU, K.; YOSHIDA, N.; NAKAMURA, T. Three-dimensional cephalometry using helical computer tomography: measurement error caused by head inclination. Angle Orthod., Appleton, v. 72, no. 6, p. 513-520, Dec. 2002.

14. WERTZ, R. A. Skeletal and dental changes accompanying rapid midpalatal suture opening. Am. J. Orthod., St. Louis, v. 58, no. 1 , p. 41-66, July 1970.

Endereço para correspondência

Pollyana Marques de Moura

Av. Dom Luiz, $n^{\circ} 1233$, Sala 301- Aldeota

CEP: 60.160-230 - Fortaleza/CE

E-mail: pollyanamoura@gmail.com 